REVUE DE L'INSTITUT

FRANÇAIS D'HISTOIRE

EN ALLEMAGNE

\section{Revue de l'IFHA}

Revue de l'Institut français d'histoire en Allemagne

$2 \mid 2010$

IFHA 2

\title{
Weltgeschichte et histoire globale en Allemagne
}

Un regard comparatif et ses fonctions

\section{Matthias Middell et Katja Naumann}

Traducteur : Guillaume Garner

\section{(2)enEdition}

\section{Journals}

Édition électronique

URL : http://journals.openedition.org/ifha/256

DOI : $10.4000 /$ ifha. 256

ISSN : 2198-8943

\section{Éditeur}

IFRA - Institut franco-allemand (sciences historiques et sociales)

\section{Édition imprimée}

Date de publication : 1 janvier 2010

Pagination : 247-284

ISSN : 2190-0078

\section{Référence électronique}

Matthias Middell et Katja Naumann, «Weltgeschichte et histoire globale en Allemagne », Revue de I'IFHA [En ligne], 2 | 2010, mis en ligne le 01 février 2013, consulté le 10 décembre 2020. URL : http:// journals.openedition.org/ifha/256 ; DOI : https://doi.org/10.4000/ifha.256

Ce document a été généré automatiquement le 10 décembre 2020.

(CIFHA 


\title{
Weltgeschichte et histoire globale en Allemagne
}

Un regard comparatif et ses fonctions

\author{
Matthias Middell et Katja Naumann
}

Traduction : Guillaume Garner

\section{Un regard comparatif et ses fonctions}

L'historiographie allemande a derrière elle une longue tradition d'intérêt pour la Weltgeschichte ${ }^{1}$ et les esquisses d'histoire universelle. Il ne peut s'agir ici de retracer cette histoire dans toutes ses ramifications, ni d'en donner une bibliographie complète, mais bien plutôt d'examiner les conditions intellectuelles et institutionnelles de cet intérêt actuel pour l'histoire globale. Même si un pays précis est ici pris en compte, il convient de toujours garder à l'esprit l'internationalisation croissante des échanges scientifiques. La RFA représente aujourd'hui sans aucun doute l'un des foyers de la discussion sur l'histoire globale. Cela s'explique d'abord par la recherche d'un nouveau statut dans un monde en voie de globalisation, après que les configurations de la Guerre Froide - qui avaient limité les possibilités d'action internationale des deux États allemands - ont été remplacées par d'autres. Cet intérêt s'explique également par la participation à un débat transnational inspiré par la recherche d'un nouvel ordre mondial et de ses fondements historiques - les connexions européennes, transatlantiques, postcoloniales y interagissant d'une manière complexe avec de nouvelles orientations théoriques et méthodologiques ${ }^{2}$.

À lire les présentations de la situation actuelle de l'histoire globale, qui postulent une domination écrasante de l'historiographie nord-américaine, l'existence d'une longue et fructueuse tradition d'histoire mondiale en Allemagne se trouve reléguée à l'arrière-plan ${ }^{3}$ . Se sont en effet développés deux grands récits, dans lesquels les anciennes histoires mondiales rédigées par des auteurs allemands apparaissent soit comme la préfiguration de l'intérêt actuel pour l'histoire de la globalisation, soit comme l'exemple, à ne pas suivre, antinomique d'une conception adéquate de la nouvelle histoire mondiale: 
l'universalisme hégélien, avec ses jugements erronés sur les "peuples sans histoire ", apparait ici comme la racine d'un européocentrisme qui est à dépasser et comme un obstacle sur la voie menant à une histoire globale d'inspiration multiculturelle, pour laquelle la globalisation résulte de l'interaction de peuples, de civilisations, de cultures ou de sociétés égaux, et non de la diffusion d'un modèle (ouest-)européen, ultérieurement imité par toutes les autres régions.

De manière caractéristique, Patrick Manning, dans un panorama récent des tendances en histoire globale, mentionne certes de nombreux ancêtres européens, mais après des paragraphes portant sur la philosophie de l'histoire jusqu'en 1900, sur le temps des grandes synthèses (1900-1965) et sur la fragmentation des thèmes et des interprétations de 1965 à 1990, il prend en compte, dans le chapitre consacré à l'organisation du champ de l'histoire globale après 1990, exclusivement les évolutions nord-américaines, et sa bibliographie est concentrée sur les publications états-uniennes ${ }^{4}$. Dans cette optique, la "révolution dans les études historiques", provoquée par l'histoire globale et l'intérêt accru pour l'histoire du monde non-occidental ${ }^{5}$, est évidemment localisée entre Boston et Irvine, de même que seules les évolutions affectant les universités nord-américaines sont prises en compte quand il est question du remplacement des Area Studies par les Global Studies ${ }^{6}$. En défendant la thèse d'une professionnalisation de la World History depuis une quinzaine d'années, analogue à celle de l'histoire nationale à la fin du XIXe siècle, Manning et d'autres démarquent radicalement les évolutions les plus récentes de ce qui avait précédemment existé, et distinguent les territoires (anglo-saxons) de la professionnalisation précoce des zones sous-développées?

Ces conclusions ont été, ces dernières années, fréquemment utilisées pour signaler à l'historiographie et aux sciences sociales européennes leur retard et pour inciter celles-ci à une confrontation productive avec ces tendances récentes ${ }^{8}$. Ce mécanisme pourrait être cité comme exemple typique de l'initiation d'un transfert culturel et pourrait ainsi être comparé avec les processus d'appropriation dans les deux directions qui ont toujours eu cours de part et d'autre de l'Atlantique'.

De cet état de la discussion, Jürgen Osterhammel, l'un des représentants majeurs de la pratique effective de l'histoire globale, a récemment déduit qu'il y avait certes en Allemagne une tradition de Weltgeschichte vieille de plusieurs générations, mais que la nouvelle génération, loin de s'inscrire dans sa continuité, relève bien plutôt, précisément, de ces évolutions internationales ${ }^{10}$.

Il en est résulté une situation contradictoire. L'historiographie allemande est certes l'une des plus actives pour ce qui est d'expérimenter de nouvelles approches permettant d'étudier et de présenter des phénomènes transnationaux et globaux ${ }^{11}$, mais elle ne peut cependant pas s'appuyer sur une structure institutionnelle établie. Les causes d'une telle situation sont anciennes et leur étude nécessite un bref regard sur l'histoire de la professionnalisation de la science historique en Allemagne. On verra ainsi que l'évolution des études régionales (Regionalwissenschaften) et de l'histoire extra-européenne a une signification centrale, ne serait-ce que parce que la Weltgeschichte actuelle s'est attachée à intégrer les recherches originelles sur les autres parties du monde ${ }^{12}$. Dans le même temps, il apparait que l'étude et l'interprétation de connexions mondiales a une conjoncture, correspond à des périodes d'essor des interactions sur de grandes distances ou d'évolutions de l'ordre du monde qui rendirent nécessaires de nouveaux cadres d'organisation politique et sociale du monde. De même que, dans les années 1840, dans les années 1860 ou vers 1900, l'analyse de la profondeur historique des évolutions globales 
contemporaines gagne en importance ${ }^{13}$, les deux guerres mondiales et la décolonisation ont aiguisé l'attention pour les connexions et les interdépendances à l'échelle globale ; ceci est comparable aux espoirs d'une "gouvernance globale » nés après 1989-1991, qui ont également trouvé une traduction historiographique, qu'elle soit critique ou non. La Weltgeschichte ramène ainsi vers le présent les aspects du passé que les sociétés jugent importants pour leur époque, et possède donc une portée sociale et politique qui lui est propre ${ }^{14}$. Elle ne se consacre certes pas à justifier des conceptions ou des intérêts relatifs à la politique mondiale. Mais en insérant les sociétés dans des rapports globaux, en leur assignant un espace et une signification dans ses récits sur des évolutions globales, elle constitue souvent un outil efficace pour satisfaire des intérêts diplomatiques et géopolitiques précis $^{15}$.

\section{Un regard en arrière}

Nous pouvons ici faire abstraction aussi bien de la longue tradition de l'histoire universelle comme histoire chrétienne du salut ${ }^{16}$ que des tentatives des historiens de la fin du XVIIIe siècle ${ }^{17}$, bien que le cosmopolitisme du XVIIIe siècle ait laissé des traces dans celui du début du XXe siècle ${ }^{18}$ et que ce dernier ait eu un écho chez des auteurs comme Heinz Gollwitzer ou Rudolf Vierhaus ${ }^{19}$. Au début du XIXe siècle, les universités allemandes opérèrent un tournant fondamental vers une «histoire de la patrie » qui n'était pas encore nécessairement orientée vers une unité nationale à réaliser; par ce tournant, historiens, juristes et théologiens pouvaient placer au centre de leur enseignement les particularités de l'histoire de chaque État territorial, et l'histoire de la patrie devait dans tous les cas servir à renforcer l'identification à l'espace particulier concerné. Cette confrontation, inspirée par le romantisme, avec l'universalisme de la Révolution française et avec l'unification napoléonienne de l'Europe fut le point de départ d'une évolution donnant la priorité au projet de nationalisation des sociétés, et dont une dimension essentielle était, à côté de l'intégration économique et de la consolidation politique des États nationaux, le renforcement de la nation culturelle reposant sur une langue et une tradition communes. Cette politique culturelle impliquait non seulement d'imposer l'unilinguisme, mais aussi de former des instituteurs et des professeurs (en nombre croissant à partir des années 1860) qui donnèrent toute leur force de pénétration aux grands récits de l'histoire nationale en les adaptant aux particularités locales et régionales. Ce lien chronologique et objectif entre professionnalisation de l'histoire et nationalisation de la société a été maintes fois traitée ${ }^{20}$. On en a déduit qu'aurait pesé sur l'histoire allemande l'obligation de se plier au paradigme de l'histoire nationale, et que les aspirations émancipatrices de l'histoire comparée et des courants plaidant pour une plus forte prise en compte de l'histoire de l'Europe occidentale et orientale comme de l'histoire extra-européenne auraient, face à ce paradigme, été longtemps condamnées à l'échec, ou en tout cas marginalisées. Une approche quantitative de la production de livres, des cursus et des initiatives en matière de politique de l'histoire semble confirmer cette conception.

Cette interprétation néglige cependant un paradoxe: le XIXe siècle fut caractérisé par deux tendances allant à contre-courant de la seule prise en compte de l'histoire nationale. D'une part, la Révolution française déclencha un débat de longue haleine sur les voies de l'avènement des sociétés bourgeoises, débat qui inspira la comparaison des sociétés, et le milieu du siècle fut d'autre part marqué par une intensification des interactions globales ${ }^{21}$ 
qui se distinguait de la mondialisation de l'époque moderne dans la mesure où désormais aucune société ne pouvait, sous peine de perdre sa souveraineté, se soustraire aux connexions qui se formèrent en liaison avec l'accélération et l'intensification des échanges et avec l'apparition de véritables marchés mondiaux. La nationalisation des sociétés et l'accent mis sur les États nationaux n'était donc en rien un retrait par rapport à la globalisation, mais représentait au contraire un outil jugé approprié pour affirmer sur la scène internationale la souveraineté et la compétitivité des États et pour garantir leur nécessaire intégration intérieure (eu égard aux nouvelles dynamiques sociales et aux intérêts régionaux particuliers $)^{22}$. On peut, à l'échelle mondiale, discerner entre 1840 et 1880 des efforts visant à réagir à ces défis et à redéfinir d'anciens régimes de territorialité ${ }^{23}$ - de la Révolution de 1848 en Europe à l'ère Meiji au Japon en passant par le mouvement réformateur russe de 1861, l'autonomie relative du Canada en 1868, les Caraïbes de SaintDomingue à Cuba et les États-Unis (avec la guerre civile de 1861 à 1865) étant également touchés.

Dans les années 1880 se dessine en revanche un autre modèle: les États nationaux, désormais consolidés et dotés d'« espaces complémentaires » coloniaux, luttèrent pour capter les profits résultant de la globalisation, en contrôlant les sources de matières premières et les débouchés, mais également pour assurer des garanties géostratégiques à leurs territoires coloniaux ou leurs empires informels. Dans cette nouvelle phase de l'histoire globale, la domination libre-échangiste de l'empire britannique fut confrontée aux concurrents européens, mais aussi à l'essor des États-Unis qui se firent les avocats de la décolonisation ${ }^{24}$.

La césure entre ces deux étapes apparaît nettement pour la science historique : alors que les principaux historiens prêtèrent d'abord leur plume à la mise par écrit de l'unité nationale, une réflexion s'amorça à la fin des années 1880 afin de davantage tenir compte de l'essor des interactions. De nouvelles alliances furent nouées avec l'ethnologie ou la géographie humaine à l'occasion des voyages d'exploration sur d'autres continents, et les États ressentirent le besoin de présenter à d'autres peuples les atouts de leur système éducatif dans lequel les historiens s'imaginaient jouer un rôle central. L'idéalisation du modèle universitaire allemand, dont un premier signe avait été la présentation de volumes luxueux lors de l'exposition universelle de Chicago en $1893^{25}$, servit aussi à attirer une clientèle étudiante internationale. Les tentatives concurrentes des institutions françaises furent observées avec méfiance ${ }^{26}$ et stimulèrent les efforts visant à affirmer la présence allemande sur un marché de la formation de plus en plus international ${ }^{27}$.

Parallèlement, il s'agissait de redélimiter les frontières de la formation historique dans les séminaires d'histoire. Les universités réagirent d'abord à l'essor des effectifs étudiants par une augmentation du nombre de chaires suivant des critères chronologiques: l'histoire ancienne, puis l'histoire médiévale se séparèrent de l'étude de l'ère moderne et contemporaine (Neuzeit). L'histoire économique et sociale reçut des impulsions de l'économie politique et contribua également à l'intérêt pour les évolutions touchant les pays étrangers. Mais après ces premières différenciations internes, l'attention fut donnée à une diversification dans l'espace: des chaires d'histoire de l'Europe occidentale (d'histoire anglaise et/ou française) furent créées dans les universités les plus progressistes en même temps que des chaires d'histoire de l'Europe orientale (d'abord consacrées surtout à l'histoire russe). Dans le même temps, les études régionales ( Regionalwissenschaften) apparurent dans des instituts spécifiques : leur point de départ fut l'étude (surtout philologique) des anciennes civilisations en Égypte, en Chine, en Inde, 
mais elles s'ouvrirent rapidement aux sociétés contemporaines de ces pays par des voyages de recherche, pendant que l'étude de l'Afrique, de l'Amérique latine était, d'emblée, plus fortement inspirée par des considérations géostratégiques, commerciales ou religieuses (à travers les missions) ${ }^{28}$.

Entre les années 1890 et la Première Guerre mondiale, la grande puissance éducative et universitaire qu'était l'Allemagne renforça son intérêt économique, politique et militaire à la production et la transmission d'un appareil de savoir qui était rien moins que régional, mais possédait au contraire une forte dimension mondiale.

Les modèles d'interprétation dominants qui, postulant la supériorité de l'Europe, avaient été retouchés aux couleurs du darwinisme social, du racisme et du nationalisme, furent fortement remis en cause quand il fallut intégrer à la fois l'ascension des États-Unis au rang de puissance mondiale (au plus tard depuis la victoire de 1898 contre l'empire espagnol) et de modèle de modernisation alternatif, et la victoire-surprise de l'armée du Japon sur celle du tsar en 1905. Par la suite, la "génération de 1890 " porta un intérêt accru à l'Amérique du Nord ${ }^{29}$, tout comme s'accentuèrent les efforts visant à intégrer les évolutions nord-américaines dans les cadres établis d'interprétation de la société ${ }^{30}$.

Dans ce contexte d'intérêt pour les évolutions globales ${ }^{31}$, il fallait décider de la place à accorder à l'étude de ces évolutions dans l'organisation des sciences. La « politique des savants » de l'Empire wilhelminien incitait les historiens à étudier les processus actuels ${ }^{32}$, mais la majorité d'entre eux privilégiait l'analyse de l'histoire nationale et de l'arrièreplan historique du comportement des concurrents européens.

Les arguments en présence sont clairement lisibles dans la controverse - portant sur l'étendue à donner à la formation en histoire extra-européenne - qui opposa l'historien Erich Brandenburg (Université de Leipzig), qui s'intéressa à l'histoire de l'impérialisme contemporain et fut, par sa collection exhaustive d'articles de journaux pendant la Première Guerre mondiale un précurseur de la publications de sources contemporaines ${ }^{33}$, à Karl Lamprecht qui plaidait pour un enseignement plus tourné vers l'histoire universelle. Pour ce dernier, ce qui était en jeu dans la différence entre une restriction à «l'histoire politique de l'Europe et des États méditerranéens» et l'extension vers "l'histoire entière de l'évolution culturelle de l'humanité " ${ }^{34}$ était la professionnalisation de l'histoire - qui était précisément une conquête récente - tandis que pour Brandenburg, il s'agissait de garantir le développement ultérieur de l'histoire en donnant la priorité à des objets d'actualité.

Brandenburg s'opposait à l'intégration de l'histoire de l'Asie orientale dans la formation des historiens : «Il est impossible de proposer dans une faculté d'histoire tout ce qui peut être digne d'intérêt pour un historien ; il faudrait, sinon, faire appel pour des séminaires à des juristes, des philologues, des historiens de l'art, des économistes et former une petite université annexe $»^{35}$. Selon lui, il convenait plutôt d'orienter les étudiants intéressés par l'histoire extra-européenne vers les facultés voisines spécialisées. Cependant, Brandenburg ne contestait pas la signification de l'histoire du temps présent comme point de référence central de l'enseignement et de la recherche. Son argument, selon lequel l'histoire ne peut, en raison de sa méthode, étudier que des États et des administrations développées, car elle ne possèderait pas des outils adaptés à la critique des sources non écrites, se révéla être ambivalent, parce que le partage simplificateur du monde entre civilisation européenne et retard extra-européen devint de moins en moins crédible, et que l'intérêt pour l'activité des États concurrents dans les colonies revêtit une importance existentielle. 
Lamprecht ne pouvait, en revanche, pas comprendre pourquoi les formes d'institutionnalisation traditionnelles devaient être maintenues et en appela à son succès public, à sa reconnaissance internationale pour attester du niveau que pouvaient atteindre des travaux d'histoire mondiale. Il fit valoir l'expérience accumulée par l'histoire culturelle (Kulturgeschichte) dans l'exploitation des témoignages matériels et plaida pour une collaboration étroite avec les disciplines proches qui exploitaient depuis longtemps ces sources. Il se savait en harmonie avec des efforts similaires accomplis à Hambourg, Francfort-sur-le-Main et Kiel, où les projets de création d'universités ou de l'Institut Royal pour le Commerce Maritime et l'Économie Mondiale soulignaient également le besoin d' " histoire de l'outre-mer $"^{36}$. Il n'est donc pas étonnant que ces lieux aient entretenu des échanges intenses pour savoir si les infrastructures établies étaient favorables à cette nouvelle orientation ou s'il fallait envisager des instituts de recherche d'un type nouveau et des chaires interdisciplinaires ${ }^{37}$.

Au total, il apparaît qu'au tournant des XIXe et XXe siècles, de nombreux historiens allemands - certes minoritaires - étaient à la recherche d'une nouvelle conception de l'histoire qui tiendrait compte du besoin de points de repère face à une nouvelle vague de globalisation. L'intérêt des étudiants était considérable, comme le montre l'essor rapide des effectifs de l'Institut für Kultur- und Universalgeschichte fondé à Leipzig par Lamprecht en 1909, où plus de 300 étudiants étaient inscrits - soit autant que dans la faculté d'histoire voisine. L'approche qui y était mise en œuvre était tournée autant vers la comparaison que vers l'étude des interactions, et elle prit le pas sur les anciens modèles d'histoire universelle européocentrée. L'état de la recherche nous parait, aujourd'hui, évidemment insuffisant pour une telle conception, mais cela n'en constitua pas moins des tentatives cohérentes d'adaptation de la science historique aux nouvelles exigences auxquelles la globalisation confrontait une puissance mondiale comme le Kaiserreich.

Il serait cependant trop facile de ne considérer que les quelques lieux où se formèrent des instituts qui établirent un enseignement proche des conceptions actuelles de l'histoire globale. Car dans le même temps, les instituts d'histoire traditionnels poursuivirent leur expansion avec des chaires d'histoire des autres pays européens, et surtout, de plus en plus d'étudiants choisirent l'option indiquée par Brandenburg dans son débat avec Lamprecht : ils se consacrèrent simultanément à l'histoire et aux études régionales et profitèrent ainsi de la professionnalisation de la sinologie, de l'indologie, des études africaines, latino-américaines, japonaises ou est-européennes ${ }^{38}$. À la différence de nombreux autres systèmes universitaires, dans lesquels les étudiants choisissaient une seule matière ou recevaient une introduction rapide à la diversité des savoirs enseignés dans un cours pluridisciplinaire (comme dans le College états-unien), l'organisation des études dans les universités allemandes permettait aux étudiants de choisir les cours les intéressant. Cependant, les renseignements biographiques donnés par les historiens à la fin de leurs études ${ }^{39}$ montrent qu'ils furent toujours plus nombreux, à partir de la Première Guerre mondiale, à suivre des enseignements dans les études régionales et les études slaves (Slawistik).

L'intérêt pour la Weltgeschichte fut donc satisfait dans les universités allemandes suivant deux voies. La première - quantitativement moins significative, mais attachée à la cohérence du programme de formation - était la création de nouveaux cursus en histoire universelle ou en sociologie historique, dans lesquels des spécialistes des disciplines anciennes collaboraient avec ceux des sciences sociales et des études régionales alors en émergence. La seconde, plus fréquente, consistait à développer les études régionales dans 
des instituts autonomes et à laisser les étudiants combiner les formations en histoire avec celles des Area Studies ou des disciplines philologiques ${ }^{40}$.

Les autorités universitaires reconnurent le nouveau statut des études régionales et s'efforcèrent à la fois de les développer de manière coordonnée et de mettre l'expertise nouvellement acquise «au service de l'affirmation bien comprise de la nation et du rapprochement entre les nations $»^{41}$. Les premiers efforts visant à fonder, sur le modèle français de l'École libre des sciences politiques, une École supérieure pour la politique étrangère échouèrent en 1917 devant le refus des Länder de concéder au Reich des compétences particulières, et ces questions tombèrent dans le domaine de compétence des États fédérés. Mais le sujet était passé à l'ordre du jour ${ }^{42}$, et dans le débat entre l'islamologue Carl Heinrich Becker, futur conseiller du ministre prussien de l'Éducation, et le pédagogue Eduart Spranger, ce dernier résumait en 1917: «En même temps que s'achevait tardivement notre existence nationale, nous sommes, presque sans le remarquer, entrés dans l'ère de l'économie mondiale et donc de la politique mondiale. C'est pourquoi notre prochaine mission n'est pas du tout de satisfaire aux aspirations coloniales et impérialistes de l'ère de la politique mondiale, mais de rattraper ce qui nous a jusqu'à présent manqué: une éducation du peuple aux relations avec les autres peuples $»^{43}$. Dans les années suivantes, Becker suivit une politique de développement systématique des études sur les pays étrangers (Auslandsstudien), et il s'assura pour cela de l'appui non seulement des représentants des matières à soutenir, mais aussi des universités en Prusse et hors de Prusse, auxquelles il offrit une nouvelle légitimation en leur accordant une importance nationale dans la production concertée de savoirs sur le reste du monde.

Bien que cette politique ait manifesté une certaine continuité avec la situation d'avant 1914, la Première Guerre mondiale représenta à plusieurs égards une césure importante, comme dans d'autres domaines. C'est une tendance générale: les processus de transformation peuvent s'accélérer dans des situations exceptionnelles telles que les guerres, l'expérience de l'utilisation des ressources civiles pour des buts de guerre renforçant la disposition aux réformes, que ce soit sous des auspices nouveaux ou selon des directions précédemment tracées ${ }^{44}$. Dans beaucoup de pays, la guerre renforça les arguments en faveur d'une réorganisation rapide des universités et d'un renforcement des sciences nationales ${ }^{45}$.

La défaite enterra d'abord les ambitions coloniales et réduisit fortement le rôle de l'Allemagne comme grande puissance dans la politique mondiale. L'intérêt pour la globalisation fut désormais motivé par la perspective de la revanche, du recouvrement d'une position de puissance politique et donc d'une remise en cause de l'ordre issu de l'après-guerre, et moins par l'idée d'une responsabilité à assumer dans le fonctionnement de la politique mondiale.

Sous le coup d'une situation extrême ressentie comme ouverte, et dont la révision n'était pas poursuivie par les seuls conservateurs, les recherches entamées avant 1914 sur les Allemands de l'étranger se radicalisèrent dans le sens d'une ethnicisation des conflits qui y étaient liés et dans le sens d'une instrumentalisation de ces communautés comme " cinquième colonne " pour de futures aventures militaires. Ce furent précisément ces recherches qui permirent la jonction entre des approches en termes de Weltgeschichte et la Volksgeschichte ${ }^{46}$ qui gagna en importance à partir de 1924 et offrit un nouveau paradigme de l'histoire nationale, plus porteur pour une politique révisionniste que l'ancienne approche centrée sur l'État. 
À partir de 1916, les sciences humaines allemandes opérèrent une entrée en guerre qui préfigura l'utilisation des études sur les pays étrangers dans les années 1930-1940 ${ }^{47}$. Dans l'occupation de la Belgique et l'avancée vers la Balkans comme dans l'inspiration pour le Djihad afin de saper la domination coloniale britannique au Proche-Orient ${ }^{48}$, les linguistes, les géographes et les spécialistes des sciences de la culture jouèrent de plus en plus un rôle de conseillers pour l'armée, et c'est sur la base de ces expériences que se développa, dans les années 1920, une nouvelle orientation des études régionales qui avaient cependant, déjà avant 1914, influencé les sciences coloniales. À la place d'une approche comparative, qui était directement compatible avec l'enseignement de l'histoire mondiale, les études sur les pays étrangers s'ouvrirent à une exploitation à des fins politiques concrètes, ce qui se traduisit par une spécialisation des différents lieux dans l'étude de parties du monde précises: l'empire britannique devait être étudié à Kiel, l'empire russe à Breslau et Königsberg, l'Amérique, le Proche et le Moyen Orient à Berlin, les Balkans à Leipzig, la France à Bonn et l'Italie avec la Méditerranée occidentale à Munich ${ }^{49}$. Cette proposition de Spranger, qui, sans être intégralement appliquée, avait pour elle de regrouper de manière commode les principaux axes de recherche existants, déchira les configurations établies avant-guerre qui, par un vaste déploiement des études régionales à Berlin et Leipzig, permettaient un comparatisme étendu aux dimensions de l'histoire universelle.

La Weltgeschichte ne disparut pas de l'enseignement, d'abord parce que les institutions fondées avant 1914 et les pionniers de l'histoire universelle reposant sur l'esprit de la Weltpolitik ne disparurent pas, mais l'apogée de sa force d'innovation intellectuelle et de sa résonance sociale était manifestement derrière elle. La Propyläen-Weltgeschichte, éditée par Walter Goetz à la fin de la République de Weimar, reste digne de mention. Ce libéral rechercha certes vainement, encore dans la seconde moitié des années 1920, la coopération de partenaires occidentaux, mais dans l'interprétation de l'histoire contemporaine, cette histoire parvint à se rattacher au "grand récit» qui jugeait l'évolution politique à l'aune de la démocratie parlementaire, et faisait de cette dernière une conséquence des révolutions des XVIIIe et XIXe siècles. Selon Goetz, l'évolution de l'Allemagne était compatible avec l'ascension de la civilisation occidentale depuis que la république avait succédé au Kaiserreich. Une telle conception - dont la marginalité fut avérée quand l'historien et ancien député du Reichstag fut contraint à l'éméritat juste après la prise du pouvoir par les nazis - justifia une attention soutenue pour les évolutions en Europe occidentale et offrit en même temps peu de raisons d'étudier l'histoire africaine, asiatique ou sud-américaine.

Les années 1920 préfigurèrent l'évolution postérieure à l'effondrement du IIIe Reich de deux manières. La politisation des études sur les pays étrangers s'était fortement accrue sous la dictature nazie ${ }^{50}$ et n'offrit aucun point d'appui pour la lente reconstruction des études régionales de l'après-guerre, mais encouragea au contraire leur orientation philologique, au contraire du produit de la Guerre Froide qu'étaient les Area Studies, nées aux Etats-Unis et vite tournées vers les problématiques des sciences sociales ${ }^{51}$. L'histoire se concentra en revanche sur l'explication du "Sonderweg allemand» et se référa au modèle des régimes démocratiques mis en place en Europe occidentale et en Amérique du Nord $^{52}$. Dans les années 1960, le grand récit de Walter Goetz fut repris par Golo Mann dans la réédition de son histoire mondiale. De même, les histoires mondiales ultérieures, comme celle de l'éditeur Fischer (1965-1980) étaient (notamment dans le choix des auteurs) tournées vers les interprétations des historiens britanniques et français, mais 
sans abandonner le cadre interprétatif établi. Le fait que les sociétés allemandes ne furent, à la différence de leurs voisines occidentales, qu'indirectement confrontées à la décolonisation fit sentir ses effets. Dans ces circonstances, une tradition autonome de l'histoire impériale comme creuset des différentes Area Studies ne put se développer ${ }^{53}$. La priorité était au contraire donnée à la recherche d'explications de l'avènement et des crimes du national-socialisme - ceci étant accentué par la concurrence entre les deux historiographies allemandes ${ }^{54}$.

Les conditions étaient donc tout sauf favorables à la poursuite d'un modèle d'enseignement non intégré de la Weltgeschichte : les études régionales et l'historiographie suivaient des voies divergentes, le paysage institutionnel de la science historique fut réorganisé et les conséquences de l'exode de chercheurs internationalement reconnus après 1933 furent à court terme difficiles à compenser. En RFA, la prise de conscience de la défaite et des crimes dont les Allemands avaient à répondre, mais également les possibilités d'action diplomatique restreintes dans le cadre de la Guerre Froide, rendirent prioritaires l'« éducation à la démocratie » et la formation d'une société se définissant comme postnationale.

En RDA, les continuités par rapport à l'avant-guerre furent bien moins importantes. Alors que la recherche d'une légitimation historique du « premier État ouvrier et paysan sur le sol allemand» encouragea l'histoire nationale, la tradition internationaliste du mouvement ouvrier et communiste offrit un point de départ à une nouvelle Weltgeschichte se réclamant de la conception holiste de l'histoire élaborée par Marx. Le rapport de forces entre ces deux orientations dans les différentes universités avait de nouveau plus à voir avec des continuités institutionnelles que ne pouvait le faire croire la rhétorique du nouveau départ ${ }^{55}$.

L'intérêt pour les problématiques de la Weltgeschichte fut encouragé d'abord par la coopération avec les chercheurs marxistes d'Europe orientale et occidentale dans l'étude des processus de transition et dans l'insertion de la région de la mer Baltique et de l'Europe centrale et orientale dans la Weltgeschichte. Dans les années 1950, les historiens est-allemands et tchécoslovaques, avec des collègues occidentaux, furent sensibles aux attraits des recherches sur la Hanse et la mer Baltique pour l'histoire universelle ${ }^{56}$ dans le cadre de la Hansische Arbeitsgemeinschaft qui avait été fondée en 1955 à l'initiative du médiéviste de Leipzig Hans Sproemberg. Ce dernier et ses alliés étaient parvenus à transnationaliser l'histoire de la Hanse et à l'étudier comme un élément d'une Weltgeschichte à dominante économique et sociale ${ }^{57}$. Sous la direction de Sproemberg, ce groupe de travail devint un lieu de coopération internationale, tout comme le Leipziger Arbeitskreis mittelalterlicher Historiker qu'il avait également fondé, et sa chaire devint vite un lieu central des discussions sur la mer Baltique ${ }^{58}$. Cette intention d'appréhender le commerce baltique dans ses rapports globaux était toujours présente dans les années 1960, d'autant plus que « les problèmes internationaux du commerce de la mer du Nord et de la mer Baltique... [tout comme] la position du commerce hanséatique dans le cadre du commerce mondial $»^{59}$ étaient devenus des thèmes centraux des recherches sur la Hanse. Mais cette coopération et cette approche transnationale cessèrent avec la construction du Mur, et la Hanse fut de nouveau étudiée dans une optique nationale, jusque dans les années 1990 et les premiers essais d'une étude de cet espace en termes d'histoire globale ${ }^{60}$

D'autre part, et de manière plus concluante, la Weltgeschichte se développa en réaction aux défis politiques et scientifiques lancés par la décolonisation, des universitaires est- 
allemands ayant tôt participé à la mise en place de systèmes d'éducation dans les nouveaux États nationaux d'Afrique, d'Asie du Sud-Est et du Proche-Orient ${ }^{61}$. Parce qu'elle recherchait une reconnaissance internationale et parce qu'elle participait à l'aide au développement menée par les pays de l'Est, la RDA avait un besoin croissant d'experts pour la coopération politique, diplomatique, économique et culturelle avec les mouvements de libération nationale, et comme elle cherchait en outre à ancrer les évolutions récentes des États aspirant à l'indépendance dans sa conception du monde et de l'histoire, les études régionales bénéficièrent d'un soutien massif.

Des historiens comme Walter Markov jouèrent un rôle central dans la formation d'universitaires présents à la fois dans les universités locales, la recherche sur le terrain et la politique de développement, et les universités de RDA se présentèrent aux nouveaux chercheurs des États du Tiers Monde comme des lieux de formation scientifique. Ainsi, dans les années 1960, une nouvelle chance se présenta de reconstruire le modèle antérieur à 1914 incluant comparaison des civilisations et Area Studies, et dans la première phase de la IIIème réforme universitaire (jusqu'au printemps 1968), des plans furent élaborés pour créer des institutions qui devaient lier dans la recherche et l'enseignement les études régionales et une histoire mondiale d'orientation marxiste ${ }^{62}$.

Cette floraison s'acheva cependant dans la seconde phase de la réforme (1968-1971) pour deux raisons. D'une part, le manque de ressources imposa l'arrêt de l'expansion des études régionales et la concentration des moyens disponibles. Ainsi fut de nouveau anéantie la possibilité de mener en un ou plusieurs lieux une comparaison des différentes Area Studies, au lieu de quoi chaque université se vit confier l'étude d'une région du monde. La concentration des études asiatiques à Berlin, des études africaines et procheorientales à Leipzig, des recherches sur l'Amérique latine à Rostock ne fut qu'en partie appliquée, après une longue résistance des universités. En outre, le lien entre histoire et études régionales fut, à l'exception des recherches sur l'Amérique latine à Leipzig, largement rompu par la création d'instituts spécifiques pour les différentes Area Studies, et il ne fut plus tard renoué que pour les pays dits du Tiers Monde dans un "Conseil central des études africaines, asiatiques et latino-américaines ». Le rejet de l'histoire extra-européenne hors des instituts d'histoire fut ainsi de nouveau confirmé. Les chances de réaliser une convergence des compétences, qui auraient pu résulter du paradigme commun du marxisme comme théorie sociale, furent manquées, parce que les spécialistes des études régionales voyaient la séparation d'avec les «disciplines-mères » comme la preuve de leur prestige croissant, et parce que la direction politique du pays considérait à raison - le potentiel d'une histoire globale fondée empiriquement comme une menace sur son hégémonie intellectuelle et privilégia le classique "divide et impera ». À voir la prudence manifestée dans les années 1970 par les protagonistes d'un renouveau de l'histoire mondiale appliqué à l'histoire comparée des révolutions quand il s'agissait d'étudier le XXe siècle, on comprend à quel point était étroite la marge de manœuvre que leur laissait le conflit prévisible entre histoire globale et orthodoxie marxiste-léniniste, là où le pas de l'opposition à la doctrine officielle n'était pas franchi ${ }^{63}$. Alors que le projet d'un vaste institut d'histoire mondiale et d'études régionales ne put être réalisé, les tentatives ultérieures visèrent à appliquer localement ce programme dans l'enseignement et à le généraliser à l'aide d'un manuel utilisé dans toutes les universités est-allemandes. Ceci permit au moins d'établir dans les années 1980 un dialogue avec les spécialistes d'histoire globale des États-Unis ${ }^{64}$. Déjà auparavant, Walter Markov et d'autres historiens 
est-allemands avaient apporté une contribution substantielle aux débats internationaux lors des sessions du CISH (en particulier à Vienne en 1965 et à Moscou en 1970) ${ }^{65}$.

En RFA, les effets de la décolonisation furent également sensibles, et favorisèrent un essor des recherches historiques sur l'Afrique et l'Amérique latine ${ }^{66}$. Une nouvelle génération chercha à échapper au primat de l'État national allemand et de l'histoire (ouest-) européenne, et obtint le rattachement de plusieurs chaires d'histoire de l'Amérique latine et de l'Afrique aux séminaires d'histoire (ainsi à Berlin, Hambourg, Bielefeld, Eichstätt, Cologne, etc.), pendant que beaucoup de chercheurs spécialisés dans l'étude des problèmes du Tiers monde se consacrèrent à l'aide au développement et se retirèrent du monde universitaire.

Étant rapprochés institutionnellement, les cursus de formation entretinrent entre eux une proximité plus grande que cela n'avait été le cas dans le cadre de la division du travail entre instituts d'histoire et d'études régionales. Une intégration effective dans le sens de l'histoire mondiale resta cependant une tentative marginale d'historiens tels qu'Ernst Schulin à Fribourg ${ }^{67}$, qui regroupa autour de lui un petit cercle d'élèves ${ }^{68}$ qui, plus tard, stimulèrent un nouveau type d'enseignement de l'histoire mondiale, principalement par les cours de l'Université à distance de Hagen ${ }^{69}$. Une présentation globale récente du colonialisme et de la globalisation peut être considérée comme un produit direct de cette tradition intellectuelle et institutionnelle ${ }^{70}$.

L'absence d'intégration des études régionales dans l'histoire globale ne s'explique donc pas par un manque de compétences. Sans aucun doute, la dotation en personnel des Area Studies n'a pas suivi en RFA l'expansion constatée aux États-Unis, en France ou en GrandeBretagne $^{71}$. Cela se traduit par une moindre différenciation des champs de recherche et des programmes d'enseignement qui mettent en avant l'expertise de détail dans leur conception de la science. L'influence réduite qui en résulte sur les débats publics et l'enseignement secondaire a été examinée récemment ${ }^{72}$. Cette (prétendue ?) faiblesse n'explique cependant rien par elle-même. Ce sont bien plutôt des facteurs externes qui ont joué un rôle décisif. Après une période d'intérêt marqué pour les problèmes du Tiers Monde dans les années 1960, les études régionales et l'histoire mondiale souffrirent du primat accordé en RFA (tout comme en RDA) aux questions intérieures et européennes.

La politique mondiale étant l'apanage des grandes puissances, les deux États allemands s'intéressèrent avant tout à la coexistence pacifique et cherchèrent à minimiser les conséquences pour leurs propres sociétés de la course aux armements et des conflits sur les autres continents. Face à une demande réduite, la priorité pour les études régionales fut de protéger leurs moyens, notamment parce que la suppression d'une ou deux chaires pouvait menacer la spécialisation d'une université dans l'étude d'une région du monde. C'est pourquoi ces sciences fragiles furent qualifiées de manière peu flatteuse de " matières en voie de disparition ».

La réunification allemande ne profita pas non plus aux Area Studies et à l'histoire mondiale. Critiqués pour avoir mis leurs instituts richement dotés en personnel au service de finalités politiques, les spécialistes des études régionales répondirent en insistant sur les racines avant tout philologiques de leur discipline : en soulignant leur expertise sur les questions linguistiques, ils assurèrent leur existence, mais au prix d'une marginalisation partielle.

Ce n'est qu'avec les réactions des sciences humaines aux défis de la mondialisation que la situation s'est radicalement modifiée : un dialogue entre les spécialistes de l'Afrique, de l'Asie et de l'Amérique latine et les historiens qui voient dans l'histoire globale un moyen 
de comprendre la mondialisation s'est instauré ${ }^{73}$. La place croissante du financement de la recherche accordé après l'évaluation des pairs a facilité des alliances transdisciplinaires. Les projets portés par des chercheurs en histoire, sciences sociales, géographie, sciences de la culture (Kulturwissenschaften) afin d'étudier les interactions globales connaissent un essor qui est, de manière révélatrice, particulièrement massif dans le domaine de la formation doctorale ${ }^{74}$.

La pression de la recherche internationale joue certes un rôle, mais ces changements ne s'expliquent pas sans l'intérêt public pour les rapports entre les processus intérieurs et des conflits apparemment éloignés, ni sans la prise de conscience de ce que l'Allemagne n'est, sur le plan scientifique, pas préparée à son nouveau rôle de puissance politique mondiale.

\section{Un regard sur la situation actuelle}

Depuis le milieu des années 1990, le poids des facteurs institutionnels dans la politique universitaire allemande s'est quelque peu réduit. Alors que depuis 1945, les moyens financiers pour la recherche et l'enseignement étaient versés par les ministères aux universités qui les reversaient aux instituts ou aux chaires, les subventions directes de l'État sont, depuis quinze ans, restées constantes, si bien que leur partie qui pouvait être librement consacrée à de nouveaux champs de recherche a diminué. Les universités furent donc, plus qu'avant, tributaires des fonds pour la recherche fournis par la Deutsche Forschungsgemeinschaft (DFG), par les aides aux programmes thématiques de recherche des différents ministères fédéraux et par diverses fondations. La politique de ces pourvoyeurs de fonds a, beaucoup plus que celle des instituts d'histoire, tenu compte des tendances internationales, si bien que les programmes d'histoire globale ont été davantage soutenus que cela aurait été le cas dans le cadre des structures universitaires traditionnelles. Des écoles doctorales (Graduiertenkollegs) et des groupes de jeunes chercheurs se sont formés et ont ainsi permis à la nouvelle génération de participer aux débats hors d'Allemagne sans tenir compte des hiérarchies universitaires allemandes. Cette évolution est cependant à double tranchant: elle a sans aucun doute facilité de nouvelles approches, mais elle n'oblige pas ces dernières à s'institutionnaliser, si bien que des projets prometteurs risquent d'avorter ou de migrer à l'étranger.

Il règne aujourd'hui un étonnant consensus favorable à l'histoire globale, comme cela est manifeste dans les décisions sur des demandes de financement (qui, dans les fondations, sont toujours prises ou fortement influencées par des rapporteurs représentant la discipline historique), ainsi que dans la sélection des thèmes des congrès biennaux des historiens allemands ${ }^{75}$.

Dans le même temps, les conditions dans les universités allemandes sont inégalement favorables à l'apparition de cursus en histoire globale. De grandes universités, comme Munich, Francfort-sur-le-Main, Tübingen, Bonn n'ont pas de chaire d'histoire de l'Asie, de l'Afrique ou de l'Amérique latine, tandis que d'autres, telles Münster ou l'université pionnière que fut Bielefeld, ne disposent que d'une chaire d'histoire des régions extraeuropéennes. La refondation prometteuse à Erfurt, qui cherchait à réaliser un projet d'études régionales comparées au sein des cursus historiques, est confrontée, dans un contexte d'économies forcées, à la suppression de chaires qui auraient offert des conditions très favorables à la réalisation d'un programme d'histoire globale pour des étudiants de master. Ce sont donc avant tout l'université privée Jacobs de Brême ${ }^{76}$ - à 
l'enseignement intégralement en anglais - et le programme de Global Studies de Leipzig ${ }^{77}$ qui proposent l'histoire globale comme enseignement établi aux étudiants de master.

Les conditions institutionnelles semblent beaucoup plus favorables en ce qui concerne la formation des doctorants. Ces dernières années ont été marquées par l'essor en Allemagne de programmes de formation doctorale dans lesquels des représentants de plusieurs disciplines forment ensemble des doctorants travaillant sur des thèmes divers. Ce format a été introduit il y a vingt ans avec les écoles doctorales de la $\mathrm{DFG}^{78}$ et s'est depuis développé dans beaucoup d'universités. Il offre d'excellentes conditions pour la coopération entre Area Studies, histoire, sciences sociales et sciences de la culture, et a permis l'émergence de nombreux programmes qui peuvent être rattachés au domaine de l'histoire globale ${ }^{79}$.

Des instituts de recherche extra-universitaires participent souvent à de tels groupements. Parmi les centres allemands de recherche en histoire globale, dont l'orientation historique est variable selon les cas, on compte l'Institut allemand d'aide au développement à Bonn ${ }^{80}$, le German Institute for Global and Area Studies (anciennement Institut allemand de l'Outre-mer) à Hambourg ${ }^{81}$ qui est engagé dans plusieurs coopérations avec l'université, et la " Fondation science et politique » à Berlin ${ }^{82}$. On peut aussi y rattacher le Zentrum Moderner Orient à Berlin ${ }^{83}$, qui fut d'abord l'un de ces centres en sciences humaines fondés à la suite de la réunification, soutenu par la DFG, et qui bénéficie d'un financement à moyen terme par le Ministère Fédéral de l'Éducation et de la Recherche $(\mathrm{BMBF})^{84}$. Il convient de mentionner, pour le domaine de l'histoire stricto sensu, l'Institut Historique Allemand de Washington qui, par ses nombreuses journées d'études, remplit sa fonction de mise en relation des débats allemands et nordaméricains, et qui, après une réflexion menée en commun avec l'Institut historique Allemand de Londres sur la tradition et l'évolution de la discipline ${ }^{85}$, s'est récemment tourné vers les différentes dimensions de l'histoire globale de la Guerre Froide ${ }^{86}$.

Dans les universités allemandes, la recherche sur les processus de globalisation et de transnationalisation est surtout organisée autour des doctorants. Dans les groupes de recherche les plus importants, les Sonderforschungsbereiche (SFB - programmes transversaux de recherche), le sujet n'occupe pas une place centrale. Cependant, trois SFB en sciences humaines et sociales s'intéressent à ce champ. Le SFB 536 "modernisation réflexive - analyse de la transformation de la modernité industrielle " à Munich mobilise depuis 1999 des compétences en sociologie, psychologie sociale, sciences politiques, histoire et philosophie pour savoir «si les sociétés du XXIe siècle peuvent encore être comprises avec les concepts des XIXe et XXe siècles ${ }^{87}$. Selon la thèse centrale du SFB, les changements observables dans les sciences, la politique, l'économie et la société peuvent et doivent être interprétés comme une «rupture structurelle » au sein de la modernité, rupture qui est étudiée comme une transition de la "première » à la "seconde " modernité, ou de la modernité « simple » à la modernité « réflexive »"

Considérant avant tout la forme sous laquelle se présente l'État, le SFB 597 «l'État en mutation » a pour objectif d'étudier «si et de quelle manière l'État national classique s'est modifié depuis les trente dernières années sous l'effet de la pression exercée par la globalisation ou par la libéralisation ${ }^{89}$. Cette pression a été identifiée, dans la première phase du SFB, principalement mais non exclusivement aux multinationales. Cette conception rejoignait le débat sur une prétendue «fin de l'État national » ${ }^{90}$. Un développement de cette problématique centrale a été poursuivi par le SFB 700 "gouvernance dans les espaces faiblement étatisés: de nouvelles formes de 
gouvernement?» à la Freie Universität Berlin ${ }^{91}$. Il étudie comment et dans quelles conditions la "gouvernance » en matière de pouvoir, de sécurité et de bien-être a été mise en œuvre dans les espaces faiblement étatisés, et les problèmes qui en résultèrent ${ }^{92}$.

Le SFB/Transregio 15 "Gouvernance et efficacité des systèmes économiques ", qui a été créé entre les universités de Berlin (Humboldt Universität, Freie Universität), de Bonn, de Mannheim, de Munich, le Zentrum für Europäische Wirtschaftsforschung (Mannheim) et le Max-Planck-Institut zur Erforschung von Gemeinschaftsgütern (Bonn), s'intéresse également aux changements induits par la globalisation ${ }^{93}$. Avec un concept de gouvernance différent de celui du SFB 700, il étudie les effets que les changements de l'économie mondiale des années 1990 ont eus sur les tentatives de régulation étatique et le comportement des entreprises.

Les groupes de chercheurs de la DFG (FOR) constituent une autre forme d'organisation et de soutien à la recherche collective. Le FOR 517 « Compétitivité internationale et aptitude à l'innovation des universités et de organisations de recherche - de nouvelles formes de gouvernance » du Forschungsinstitut für öffentliche Verwaltung de Spire (2003-2009) réagit aux pressions exercées sur les institutions de recherche universitaires et extrauniversitaires ${ }^{94}$. Ses recherches sont centrées sur le Change Management des institutions de recherche dans un environnement transnational en pleine mutation.

Le FOR 955 «Acteurs de la globalisation culturelle 1860-1930 » fut créé à l'été 2008. Ce groupe tourné vers l'histoire et la comparaison régionale réunit des chercheurs de Berlin, Hambourg, Edimbourg, Florence, Brême et Zurich. Alors que les formes d'interactions politiques et économiques constituent un thème dominant, il s'agit ici d'examiner les interactions culturelles vers 1900. La priorité est donnée aux acteurs sociaux et aux perspectives extra-européennes, et il s'agit avant tout de déterminer si les différentes formes d'appropriation culturelle ont pu représenter un point de départ pour la formation de "modernités multiples ». Par hypothèse, il serait également possible de reconstituer une cartographie des connexions culturelles à l'échelle globale qui tiendrait compte des différentes dynamiques régionales ${ }^{95}$.

Au-delà des SFB et des FOR, la majeure partie de la recherche collective sur la globalisation est menée dans le cadre des écoles doctorales (GK) soutenues par la DFG. Le GK 844 « Société mondiale - mise en place et représentation de la globalité » (Bielefeld) se fonde sur sa tradition, très marquée par la théorie des systèmes de Niklas Luhmann ${ }^{96}$ - de 2003 à 2007, le GK s'intitulait d'abord «concepts du monde et modèles structurels globaux». Des sociologues, des politistes, des historiens, des théologiens et des chercheurs en anthropologie sociale ont fondé leur programme sur l'hypothèse selon laquelle " la société moderne est caractérisée par un rapport global », à savoir « la société mondiale $\aleph^{97}$. La globalisation est par conséquent conçue comme une étape supplémentaire de la différenciation de la société mondiale. Initialement d'orientation macrosociologique, le GK a ensuite étudié «les microstructures dans la société mondiale ", avant de s'intéresser dans une troisième étape à «la production et la représentation de la globalité ».

Depuis 2006, le GK 1261 «Zones de rupture de la globalisation » (Université de Leipzig) analyse les processus de globalisation à travers des zones de rupture qui y naissent ${ }^{98}$. Celles-ci renvoient aux espaces, aux moments et aux arènes de la globalisation dans lesquels l'établissement de nouveaux rapports à l'espace est un enjeu de conflits ${ }^{99}$. La resegmentation du monde s'y fait particulièrement sentir, et elles sont caractérisées par la déstabilisation des anciens et l'établissement de nouveaux rapports à l'espace. Ces 
zones produisent des ressources spécifiques pour l'action sociale et culturelle. L'éventail des disciplines représentées est large, allant de la science politique et la sociologie à l'histoire, en passant par les études régionales (Afrique, Asie, Proche-Orient) et les études théâtrales.

Trois écoles doctorales se consacrent à des tendances explicitement transnationales. À Giessen, le GK 891 «Événements médiatiques transnationaux de l'époque moderne à nos jours » étudie les formes et les fonctions de la présentation médiatique d'événements-clés depuis l'invention de l'imprimerie jusqu'à l'ère globalisée de l'Internet ${ }^{100}$, et privilégie les événements ayant fait l'objet d'une attention particulière, par exemple le "printemps allemand" ou la révolution roumaine de 1989 dans sa mise en scène télévisuelle, événements qui sont interprétés comme des catalyseurs des connexions globales ${ }^{101}$. Les catégories d'« opinion publique mondiale » (Weltöffentlichkeit) et de médialité de l'histoire sont ici reprises pour les besoins de l'analyse ${ }^{102}$. Le GK 1474 "Soutien social transnational ", également créé en 2007 et sis dans les universités de Mayence et Hildesheim, incarne une forme de spécialisation transnationale ${ }^{103}$. Tourné vers les sciences de l'éducation, ce GK étudie des évolutions globales, par lesquelles « les futurs défis de l'aide sociale ne pourront plus seulement être relevés dans le cadre de structures et de relations de soutien s'inscrivant dans le cadre des sociétés et des États nationaux ». Les termes-clés sont: "Transnational Family Care", "Transnational Networks" et " Transnational Professional Support", qui sont également étudiés dans le contexte des migrations internationales et de l'activité d'organisations transnationales. L'intérêt accru pour le spatial turn dans les sciences humaines et sociales en général, qui célèbre la redécouverte de l'espace comme catégorie analytique, et pour les espaces transnationaux en particulier, s'incarne dans le GK « Espaces transnationaux » créé en 2008 à l'Université Viadrina de Francfort-sur-l'Oder. Il a été mis en place dans le contexte d'une « intensification des processus de globalisation économique, politique et culturelle », qui rend la « concentration exclusive sur les espaces-containers de l'État national de plus en plus obsolète $»^{104}$. Tout comme dans le GK de Leipzig, les multiples processus de déterritorialisation/ reterritorialisation occupent une place centrale. Du point de vue géographique, ce GK se limite à l'étude de l'Europe centrale et orientale ${ }^{105}$.

«Contact culturel et discours scientifique » constituent le thème du GK 1242 de Rostock créé en 2006. Son objectif est d'étudier empiriquement les phénomènes de contact culturel en liaison avec des réflexions sur les évolutions affectant les discours scientifiques portant précisément sur les dits phénomènes ${ }^{106}$. Ce GK réunit l'histoire, les études nord- et latino-américaines, la sociologie religieuse, la musicologie et la science politique, la période prise en compte allant de l'Antiquité au temps présent. Un GK international («Histoire et culture des métropoles au XXe siècle»), plus tourné vers l'époque contemporaine, s'est formé entre les universités berlinoises et la New York University afin de mener une comparaison entre les deux métropoles ${ }^{107}$.

Le GK 1263 «Constitution au-delà de l'État : de la communauté juridique européenne à la communauté juridique mondiale? " à l'Université Humboldt de Berlin étudie, sous un angle essentiellement juridique et en tenant compte des expériences européennes, les questions de validité du droit et d'une constitutionnalisation à l'échelle globale: «le thème central est l'étude des conséquences juridiques de l'intensification et de la mise en réseau des relations entre personnes dans un monde globalisé, et du rôle du droit comme outil de garantie de la paix entre les hommes, les peuples et les États ${ }^{108}$. 
Jusqu'en 2008, un financement avait été accordé à un GK de l'Institut de sciences politiques de l'Université de Tübingen intitulé « Défis globaux - solutions transnationales et transculturelles " centré sur l'analyse de processus de règlements des problèmes supranationaux et de la signification des valeurs et des principes face aux défis « globaux ${ }^{109}$.

On peut espérer des résultats importants pour comprendre l'intertextualité et la multimédialité de la part du GK 1369 «Inter Art » de la Freie Universität Berlin (lancé en 2006), même si les questions liées à la globalisation ne sont pas explicitement thématisées ${ }^{110}$. Le dialogue entre musicologie, études théâtrales, histoire de l'art, études littéraires et cinématographiques sur les questions de performativité, d'hybridation et de multiplication des medias s'inscrit cependant clairement en réaction à des processus désignés par les sciences sociales comme relevant de la globalisation.

Il existe en outre de nombreux programmes doctoraux non concernés par le soutien de la DFG et traitant de la globalisation. Le collège doctoral « Global Social Policies and Governance ", implanté à l'Université de Kassel et financé par les fondations Heinrich-Böll et HansBöckler, s'intéresse aux asymétries économiques, écologiques et politiques croissantes entre Nord et Sud, la question centrale étant celle des réponses politiques qui peuvent être apportées à ces changements et menaces ${ }^{111}$.

Un collège doctoral a été créé à la Faculté de philosophie de l'Université de Mannheim sous le titre: «Formations du global : la globalisation du point de vue des sciences de la culture $»^{112}$. Un programme doctoral international «transnationalisation et régionalisation du XVIIIe siècle à nos jours » existe depuis 2001 à l'Université de Leipzig : il s'est d'abord consacré à l'étude comparée des processus de territorialisation politique, économique et culturelle au-delà de l'État national en Europe occidentale et orientale, en Amérique du Nord et du Sud, en Afrique subsaharienne, au Proche-Orient et en Asie orientale, et s'est tourné récemment vers l'expertise régionale ${ }^{113}$.

L'Université Leibniz de Hanovre représente un autre point de jonction de l'histoire et des Area Studies. Elle a été marquée par l'activité de Hans-Heinrich Nolte, principal spécialiste du système-monde en Allemagne, qui a initié une large palette de colloques et de publications touchant un public élargi.

Il convient enfin d'évoquer des universités situées dans des pays germanophones et qui ont une grande influence sur la recherche en Allemagne. L'ETH (Eidgenössische Technische Hochschule) de Zurich s'est spécialisée dans l'étude de l'Asie et de l'Europe et vient de recruter l'un des meilleurs spécialistes suisses de la société civile globale en la personne de l'indologue Harald Fischer-Tiéné. Sous l'égide de Peter Feldbauer, un réseau de coopération entre les études régionales et l'histoire a été développé à l'Université de Vienne : il rassemble des auteurs germanophones dans une série de livres consacrées aux "régions du monde », et il a en outre "exporté » le concept de cycles de conférences pluridisciplinaires vers Hanovre et Berlin ${ }^{114}$. Peter Feldbauer a également édité une autre série d'ouvrages sur les interactions globales depuis la fin du Moyen Âge ${ }^{115}$ et dirigé une histoire globale du monde en huit volumes ${ }^{116}$. Depuis 2006, l'Université de Vienne participe à un master européen de « Global Studies ».

Il existe certes relativement peu d'endroits remplissant les conditions nécessaires (diversité des disciplines et volonté de coopération entre elles) pour traduire l'essor de l'histoire globale dans des programmes d'enseignement ; néanmoins, des regroupements plus ou moins stables apparaissent dans de nombreux endroits afin de poursuivre les 
recherches et l'enseignement dans cette direction. Il ne s'agit donc aucunement d'un phénomène marginal, et le retard pris sur les États-Unis est donc à relativiser.

Les recherches sur la globalisation et la transnationalisation semblent particulièrement attractives pour les jeunes chercheurs. La densité des écoles doctorales et le grand nombre de candidatures qu'elles reçoivent en attestent. Il en résulte non seulement des apports intellectuels, mais une cohorte de chercheurs qui vivent une socialisation scientifique (en général après un master pluridisciplinaire) dans le domaine des études à forte dimension historique sur la globalisation. Cette jeune génération opère un "tournant empirique " en histoire globale, où il s'agit d'apporter une réponse fondée empiriquement à des " problèmes touchant à l'histoire mondiale $»^{117}$.

La parution de synthèses importantes, parfois en plusieurs volumes, sur des époques particulières ou sur l'histoire mondiale montre aussi l'intérêt existant pour les questions d'histoire globale. Jürgen Osterhammel et Nils Petersson ont publié une première histoire de la globalisation dans un petit ouvrage qui replace en perspective historique le diagnostic actuel d'une globalisation croissante. Ces deux chercheurs de Constance, spécialistes de la Chine pour l'un et d'histoire économique pour l'autre, étudient le développement de réseaux et d'espaces d'interactions qui traversent, selon eux, une phase d'élaboration jusque vers 1750 ; la période 1750-1880 correspond à la formation de l'économie mondiale, marquée par le libre-échange et l'impérialisme, et se termine avec l'expérience des guerres. Le chapitre sur la période postérieure à 1945 traite de la globalisation séparée pendant la Guerre Froide, dont la fin rendrait les effets de la globalisation toujours plus perceptibles, la terminologie liée à ces processus acquérant un caractère d'évidence toujours plus marqué118.

Jürgen Osterhammel a publié, quelques années plus tard, une magistrale histoire globale sur la «transformation du monde " au cours du XIXe siècle qui soutient la comparaison avec le livre de Christopher Bayly. Désireux de mesurer la validité des concepts de réseaux et d'espaces d'interaction, l'auteur traite en détail des problèmes liés à une périodisation spécifique à l'histoire globale et au traitement de l'espace comme cadre et produit de l'interaction; il étudie les transitions et les conflits entre populations sédentaires et nomades à l'époque des colonies de peuplement, les relations entre les migrations et l'intégration dans le cadre de l'État-nation, tout comme les différents aspects de l'industrialisation et ses conséquences pour le monde du travail, les hiérarchies sociales, la question énergétique, les relations entre l'homme et la nature, avant de terminer ce parcours du XIXe siècle par l'étude des questions religieuses et de la sécularisation ${ }^{119}$.

Hans-Heinrich Nolte travaille également à une histoire du monde en trois volumes, dont jusque-là deux volumes sont parus. Ils étudient la période postérieure au XVe siècle et $s^{\prime}$ inscrivent dans la lignée d'Immanuel Wallerstein ${ }^{120}$. Une entreprise, prévue en six volumes, de l'Académie des sciences de Mayence est encore plus ambitieuse; les deux premiers volumes publiés jusqu'ici visent à présenter les fondements du monde global depuis la préhistoire jusqu'au XIIe siècle ${ }^{121}$ et à proposer un nouveau récit de l'expansion de l'époque moderne ${ }^{122}$. Face à une telle quantité de faits et d'interprétations, les essais d'un format limité paraissent presque déplacés, même s'ils ont aidé, comme c'est le cas de l'essai de Dan Diner («comprendre le siècle ») ${ }^{123}$, à ouvrir des perspectives souvent négligées. 
Cette production impressionnante s'accompagne de débats vifs sur les concepts, la périodisation et les buts politiques d'un programme d'histoire globale ${ }^{124}$ qui est très diversifié et annonce dans un avenir proche une récolte abondante.

\section{NOTES}

1. NdT. : ce terme est l'équivalent de l'anglais World history, et, comme ce dernier, ne possède pas de traduction exacte en français. C'est pourquoi nous avons gardé le terme allemand 2. A. IRIYE, P.-Y. SAUNIER (dir.), Palgrave Dictionary of Transnational History, Basingstoke, 2009 ; M. MIDDELL (dir.), Transnationale Geschichte als transnationale Praxis, Leipzig, 2010.

3. Voir l'article de H.-H. NOLTE, «Zum Stand der Weltgeschichtsschreibung im deutschen Sprachraum » in : Zeitschrift für Weltgeschichte, 9 , 2008, $\mathrm{n}^{\circ}$ 2, p. 89-113.

4. P. MANNING, Navigating World History. Historians create a Global Past, New York, 2003, p. 17-121. Ce constat n'oublie pas que l'étude de Manning concerne surtout les doctorants et jeunes chercheurs états-uniens. La vision que l'histoire globale anglo-américaine a d'elle-même apparaît bien plus dans les conférences sur le thème "Mondialisation de l'histoire: concepts et méthodologies ", tenues lors du 19e Congrès International des Sciences Historiques (CISH) en 2000 , tout comme dans le panorama historiographique publié dans le premier numéro du Journal of Global History. Cf. Proceedings. Reports, Abstracts and Round Table Introductions, 19th International Congress of Historical Sciences, Oslo, 2000, p. 3-52; P. O'BRIEN, « Historiographical traditions and modern imperatives for the restoration of global history » in : Journal of Global History, 1, 2006, p. 3-39.

5. P. MANNING, Navigation ..., op. cit., p. 121-182.

6. Ibid., p. 166 suiv.

7. Id., "Methods and Materials » in: M. HUGHES-WARrington (dir.), Palgrave Advances in World Histories, New York, 2005, p. 44-63, ici p. 48 suiv., et d'autres articles de cette introduction très utile. Pour une approche évoquant également les évolutions en Asie : D. SACHSENMAIER, "Global History and critiques of western perspectives » in : Comparative Education, 42, 2006, n 3, p. 1-20.

8. Voir ainsi E. FUCHS, B. STUCHTEY (dir.), Across Cultural Borders. Historiography in a Global Perspective , Lanham 2002 ; EAD. (dir.), Writing World History, 1800-2000, Oxford 2003 ; E. FUCHS, M. MIDDELL (dir.), Teaching World History (Comparativ. Zeitschrift für Globalgeschichte und vergleichende Gesellschaftsforschung, 16,1), Leipzig, 2006.

9. Sur la réception d'un prétendu modèle allemand aux États-Unis: G. LINGELBACH, Klio macht Karriere. Die Institutionalisierung der Geschichtswissenschaft in Frankreich und den USA in der zweiten Hälfte des 19. Jahrhunderts, Göttingen, 2003; sur la soi-disante «américanisation » de l'enseignement supérieur allemand: S. PAULUS, Vorbild USA ? Amerikanisierung von Universität und Wissenschaft in Westdeutschland 1945-1976, München, 2010.

10. J. osterhammel, "Global History in a National Context: The Case of Germany" in: Österreichische Zeitschrift für Geschichtswissenschaft, 20, 2009, n² 2, p. 41.

11. Cette thèse peut être vérifiée par une quantification reposant sur des statistiques des participants aux congrès européens d'histoire globale, du nombre d'articles publiés dans des revues ou sur des sites internet spécialisés et des ateliers et colloques organisés par des institutions ou des chercheurs allemands. Voir notre exploitation des données sur le site 
spécialisé geschichte.transnational, à paraître dans le numéro 5/2010 de la revue Comparativ. Zeitschrift für Globalgeschichte und vergleichende Gesellschaftsforschung.

12. M. MIDDELL, K. NAUMANN, « Global history and the spatial turn. From the impact of area studies to the study of critical junctures of globalisation » in: Journal of Global History, 5, 2010, $\mathrm{n}^{\circ} 1$, p. 149-170.

13. M. MANN (dir.), Globale Geschichtsschreibung um 1900 (Periplus. Jahrbuch für außereuropäische Geschichte 18), Münster, 2008, sowie M. MIDDELL, Weltgeschichtsschreibung im Zeitalter der Verfachlichung und Professionalisierung. Das Leipziger Institut für Kultur- und Universalgeschichte, 1890-1990, 3 vol. , Leipzig, 2005.

14. "World history, then, is inevitably about world-making...»: A. DIRLIK, "Confounding Metaphors, Inventions of World. What is World History for ?» in : B. Stuchtey, E. FUCHS, (dir.), Writing World History 1800-2000, Oxford, 2003, p. 91-133, ici p. 92.

15. Sur les implications politiques de la World history actuelle aux États-Unis, voir J. BENTLEY, « Myths, Wagers, and Some Moral Implications of World History » in : Journal of World History, 16, 2005, $\mathrm{n}^{\circ}$ 1, p. 51-82 ; A. DIRLIK, « Performing the World. Reality and Representation in the Making of World Histor(ies)» in: Comparativ, 16, 2006, $\mathrm{n}^{\circ} 1$, p. 21-35. Sur les connexions entre World history australienne et aspirations à l'émancipation envers la Grande-Bretagne, voir M. HUGHESWARRINGTON, I. TREGENZA, "State and Civilization in Australian New Idealism, 1890-1950 » in: History of Political Thought, 29, 2008, n 1, p. 89-108.

16. H. ZEDELMAIER, « Die Marginalisierung der Historia sacra in der frühen Neuzeit » in : Storia della Striografia, 35, 1999, p. 15-26.

17. H. E. BÖDEKER, « The Debates about Universal History and National History around 1800 : A Problem-oriented Historical Attempt » in : Proceedings of the British Academy, 134, 2006, p. 135-170. Pour le contexte européen, voir S. BERGER, M. DONOVAN, K. PASSMORE (dir.), Writing National History: Western Europe since 1800, London, 1999.

18. Présentation détaillée de cette lignée : M. MIDDELL, « World Orders in World Histories before and after World War I» in: S. CONRAD, D. SACHSENMAIER (dir.), Competing Visions of World Order. Global Moments and Movements, 1880s-1930s, New York/ London, 2008, p. 97-120.

19. H. GOLLWITZER, Geschichte des weltpolitischen Denkens, Göttingen 1971 ; sur cette tradition : J. OSTERHAMMEL, " Global History in a National Context », op. cit., p. 50.

20. Pour un aperçu, voir G. G. IGGERS, Deutsche Geschichtswissenschaft. Eine Kritik der traditionellen Geschichtsauffassung von Herder bis zur Gegenwart, (1ère édit.) München, 1968; 2e édit., Wien/ Köln/ Weimar, 1997; ID., Geschichtswissenschaft im 20. Jahrhundert. Ein kritischer Überblick im internationalen Zusammenhang, Göttingen, 1993.

21. C. BRIGHT, M. GEYER, «Globalgeschichte und die Einheit der Welt im 20. Jahrhundert » in : Comparativ, 4, 1994, $\mathrm{n}^{\circ}$ 5, p. 13-45.

22. U. ENGEL, M. MIDDELL, «Bruchzonen der Globalisierung, globale Krisen und Territorialitätsregimes - Kategorien einer Globalgeschichtsschreibung » in : Comparativ, 15, 2005, $\mathrm{n}^{\circ}$ 5-6, p. 5-38. Voir l'exemple du Kaiserreich : S. CONRAD, Globalisierung und Nation im deutschen Kaiserreich, München, 2006.

23. Sur le concept de régime de territorialisation, voir C. MAIER, «Consigning the Twentieth Century to History: Alternative Narratives for the Modern Era» in: American Historical Review, 105, 2000, p. 807-831 ; Id., « Transformations of Territoriality 1600-2000 » in : G. BUDDE, S. CONRAD, O. JANZ (dir.), Transnationale Geschichte. Themen, Tendenzen und Theorien, Göttingen, 2006, p. 32-55.

24. C. TORP, Die Herausforderung der Globalisierung. Wirtschaft und Politik in Deutschland 1860-1914, Göttingen, 2008.

25. F. PAULSEN, Geschichte des gelehrten Unterrichts auf den deutschen Schulen und Universitäten vom Ausgang des Mittelalters bis zur Gegenwart (2e édit.), Berlin/ Leipzig, 1896-1897, 2 vol.; Id., Die deutschen Universitäten und das Universitätsstudium, Berlin, 1902. 
26. C. CHARLE, «L'élite universitaire française et le système universitaire allemand (1880-1900)» in: M. ESPAGNE, M. WERNER (dir.), Transferts. Les relations interculturelles dans l'espace francoallemande (18ème-19ème siècles), Paris, 1988, p. 345-358 ; ID., La république des universitaires 1870-1940 , Paris, 1994.

27. À l'arrivée d'étudiants étrangers dans les meilleures universités allemandes succéda bientôt la mise en place de bureaux d'information internationaux, de maisons d'hôtes, de programme d'échanges avec des professeurs américains, des assistants de langue français ou belges, tout comme des cours d'été internationaux, et des projets d'ambitieuses histoires des États nationaux impliquant des savants étrangers, etc. En une quinzaine d'années, l'enseignement supérieur allemand développa toutes les formes d'internationalisation qui ont, jusqu'à nos jours, montré leur efficience; voir B. VOM BROCKE, «Der deutsch-amerikanische Professorenaustausch: Preußische Wissenschaftspolitik, internationale Wissenschaftsbeziehungen und die Anfänge einer deutschen auswärtigen Kulturpolitik vor dem Ersten Weltkrieg " in: Zeitschrift für Kulturaustausch, 31, 1981, p.128-182; Id., Wissenschaftsgeschichte und Wissenschaftspolitik im Industriezeitalter. Das „System Althoff“" in historischer Perspektive, Hildesheim, 1991.

28. F.-T. GÜNTHER, Afrika- und Lateinamerikaforschung in Deutschland zwischen Kaiserreich und Drittem Reich, Leipzig, 2008; F. BRAHM, Wissenschaft und Dekolonisation. Paradigmenwechsel und institutioneller Wandel in der akademischen Beschäftigung mit Afrika in Deutschland und Frankreich, 1930-1970, Stuttgart, 2010.

29. G. KAMPHAUSEN, Die Erfindung Amerikas in der Kulturkritik der Generation von 1890, Weilerswist, 2002.

30. Sur la résonnance intellectuelle de l'expérience du voyage en Amérique de 1904 : M. MIDDELL, «Erkenntnis und Erkenntnisverlust: Karl Lamprechts Vorlesung über universalgeschichtliche Probleme 1904 in New York » in: R. HOHLS, I. SCHRÖDER, H. SIEGRIST (dir.), Europa und die Europäer. Quellen und Essays zur modernen europäischen Geschichte. Festschrift für Hartmut Kaelble, Stuttgart, 2003, p. 277-282.

31. H. BERGENTHUM, Weltgeschichten im Zeitalter der Weltpolitik. Zur populären Geschichtsschreibung im Wilhelminischen Deutschland, München, 2004.

32. R. VOM BRUCH, Wissenschaft, Politik und öffentliche Meinung: Gelehrtenpolitik im Wilhelminischen Deutschland (1890-1914), Husum, 1980 ; Id., Weltpolitik als Kulturmission : Auswärtige Kulturpolitik und Bildungsbürgertum in Deutschland am Vorabend des Ersten Weltkrieges, Paderborn, 1982 ; Id., ",Militarismus', ,Realpolitik‘ und ,Pazifismus‘. Außenpolitik und Aufrüstung in der Sicht deutscher Hochschullehrer (Historiker) im späten Kaiserreich» in: Militärgeschichtliche Mitteilungen, 1, 1986, p. 37-58.

33. C. FRIEDRICH, Erich BRANDENBURG. Historiker zwischen Wissenschaft und Politik, Leipzig, 1998.

34. Lamprecht au ministère saxon de l'éducation le 15 mars 1905 : Sächsisches Hauptstaatsarchiv, Ministerium für Volksbildung10281/ 203 PA Lamprecht, Bl. 98.

35. Lettre d'Erich Brandenburg à Karl Lamprecht du 5 mars 1905 : Sächsisches Hauptstaatsarchiv, Ministerium für Volksbildung 10281/ 203 PA Lamprecht, Bl. 104.

36. Voir pour plus de détails M. MIDDELL, Weltgeschichtsschreibung, op. cit., p. 350-351.

37. L. BRAUER, A. MENDELSSOHN BARTHOLDY, A. MEYER (dir.), Forschungsinstitute. Ihre Geschichte, Organisation und Ziele, 2 vol. , Hamburg, 1930.

38. Sur l'histoire des études régionales en Allemagne, voir S. MANGOLD, Eine „weltbürgerliche Wissenschaft". Die deutsche Orientalistik im 19. Jahrhundert, Stuttgart 2004 ; P. RABAULT-FEUERHAHN, L'archive des Origines. Sanskrit, Philologie, Anthropologie dans L'Allemagne du XIXe siecle, Paris, 2008 ; U. шоковск, German Orientalism. The Study of the Middle East and Islam from 1800 to 1945, London, 2009 ; S. MARCHAND, German Orientalism in the Age of Empire. Religion, Race and Scholarship, Washington, 2009. 
39. Par exemple au moment de la thèse de doctorat ou d'habilitation. Cette source est d'autant plus appropriée que des données sur le choix des matières annexes sont indiquées pour montrer la diversité des champs d'intérêt du candidat. Pour une carrière hors de l'université, ces données se limitaient souvent aux matières sanctionnées par un diplôme. Une étude quantitative de ces données reste à mener. Les impressions présentées ici reposent sur l'exploitation de 150 habilitations présentées dans les universités de Leipzig et Berlin.

40. Brandenburg et Lamprecht n'étaient pas ennemis, comme le montre l'ouvrage rédigé en commun pour l'histoire mondiale en six volumes éditée par Pflugk-Harttung en 1909: une histoire des relations internationales centrée sur l'Europe cohabitait avec un essai prédisant la fin de la domination européenne et esquissait une nouvelle histoire des interactions nécessitant une connaissance détaillée des cultures extraeuropéennes.

41. R. vOM BRUCH, Weltpolitik, op. cit., p. 97.

42. Pour une analyse de ce débat: G. MÜLLER, Weltpolitische Bildung und akademische Reform. Carl Heinrich Beckers Wissenschafts- und Hochschulpolitik 1908-1930, Köln/ Weimar/ Wien, 1991, chap. 5.

43. E. SPRANGER, «Denkschrift für die Einrichtung der Auslandsstudien an den deutschen Universitäten » in : Internationale Monatsschrift für Wissenschaft, Kunst und Technik, 11, 1917, n 9, col. 1031.

44. P. LUNDGREN, « Universitäten im Krieg. Skizze einer vergleichenden Zusammenfassung » in : $\mathrm{T}$. MAURER (dir.) : Kollegen - Kommilitonen - Kämpfer. Europäisch Universitäten im Ersten Weltkrieg, Stuttgart, 2006, p.353-357. Sur les temps de guerre comme périodes de novations dans le domaine des échanges scientifiques internationaux de 1914 à 1918, voir E. FUCHS, "Wissenschaftsinternationalismus in Kriegs- und Krisenzeiten. Zur Rolle der USA bei der Reorganisation der internationalen Scientific Community, 1914-1925 » in: R. JESSEN, J. VOGEL (dir.), Wissenschaft und Nation in der europäischen Geschichte, Frankfurt 2002, p. 263-284.

45. N. HAMmERSteIN, "Universitäten und Kriege im 20. Jahrhundert » in: W. RÜEGG (dir.), Geschichte der Universität in Europa, vol. 3, München p. 515-545, p. 521.

46. Voir W. OBERKROME, Volksgeschichte. Methodische Innovation und völkische Ideologisierung in der deutschen Geschichtswissenschaft 1918-1945, Göttingen, 1993 ; M. Hettling (dir.), Volksgeschichten im Europa der Zwischenkriegszeit, Göttingen 2003 ; M. MIDDELL, U. SOMMER (dir.), Historische Ostforschung und historische Westforschung im europäischen Vergleich, Leipzig, 2004.

47. Sur l'utilisation des sciences humaines par l'armée et les services secrets, voir F.-R. HAUSMANN , "Deutsche Geisteswissenschaft" im Zweiten Weltkrieg. Die „Aktion Ritterbusch“ (1940-1945), Dresden/ München, 1998.

48. W. G. schWANitz (dir.), Deutschland und der Mittlere Osten (Comparativ 14, 1), Leipzig, 2004.

49. E. SPRANGER, « Denkschrift », op. cit., col. 1052 suiv.

50. L. HACHTMEISTER, Der Gegnerforscher. Die Karriere des SS-Führers Franz Alfred Six, München, 1998.

51. Pour un témoignage contemporain: R. HALL, Area Studies. With Special Reference to Their Implication for Research in the Social Sciences (for the Committee on World Area Research Program of the Social Science Resesarch Council), New York, 1948. Cette évolution a été retracée récemment : V. L. RAFAEL, "The Culture of Area Studies in the United States » in: Social Text, 41, 1994, p. 91-111. Sur la tradition de l'histoire hors d'Europe, voir K. NAUMANN, "Die Herausbildung der Geschichtswissenschaft in den USA um 1900 und der Wandel des historischen Blicks nach Europa und Lateinamerika » in : M. MANN (dir.), Globale Geschichtsschreibung, op. cit., p. 127-152.

52. T. WELSKOPP, «Stolpersteine auf dem Königsweg. Methodenkritische Anmerkungen zum internationalen Vergleich in der Gesellschaftsgeschichte » in : Archiv für Sozialgeschichte, 35, 1995, p. 339-367; Id., « Die Sozialgeschichte der Väter. Grenzen und Perspektiven der Historischen Sozialwissenschaft » in : Geschichte und Gesellschaft, 24, 1998, p. 173-198. 
53. Voir l'analyse des différences entre la France et l'Angleterre: A. FRIEDRICHS, Die Historiographien imperialer Nationalstaaten. Großbritannien und Frankreich 1919-1968, thèse Université de Leipzig, 2010.

54. Cette rivalité se joua aussi sur la scène internationale, par exemple dans la question de l'admission des deux associations d'historiens dans le $\mathrm{CISH}$, comme dans l'International Commission for a Scientific and Cultural History of Mankind (UNESCO).

55. Le rôle central de Leipzig pour l'« histoire générale » (en RDA tout ce qui se situe hors de l'histoire nationale et du socialisme en Europe de l'Est) reposait ainsi moins sur la réception des analyses relatives à ce sujet de Marx et Lénine que sur la volonté de prolonger la tradition de Lamprecht, qui alla jusqu'à décider Walter Markov à déposer son habilitation en 1947 chez Hans Freyer, directeur de l'Institut für Kultur- und Universalgeschichte, et qui était alors la cible d'attaques politiques violentes. Voir M. Middell, Weltgeschichtsschreibung, op. cit., chap. 24.

56. K. ZERNACK, «Imperiale Erfahrungen in der Ostseeregion im Spiegel der Historiographie des 20. Jahrhunderts : Die polnische Perspektive » in: Frank HADLER, Mathias MESENHÖLLER (dir.) Lost Greatness and Past Oppression in East Central Europe: Representations of the Imperial Experience in Historiography since 1918, Leipzig, 2007, p. 70, n. 80 ; pour l'état de la recherche portant la période 1945-1990, voir : Zapiski Historyczne poświĘcone historii Pomorza i krajów Bałtyckich, 57, 1992, n 1.

57. M. MIDDELL, Weltgeschichtsschreibung, op. cit., p. 944 suiv..

58. En tant que directeur de l'Institut d'histoire générale de l'université de Leipzig, Sproemberg avait établi un séminaire régulier auquel une quarantaine d'historiens de la RFA et d'Europe furent invités entre 1953 et 1957 : V. DIDCZUNEIt, Geschichtswissenschaft an der Universität Leipzig. Zur Entwicklung des Faches Geschichte von der Hochschulreform 1951 bis zur ,sozialistischen Umgestaltung‘ 1958, thèse, université de Leipzig 1990, partie 1, p.118. Des comptes rendus des journées annuelles de ce groupe de travail ont été publiés: G. HEINTZ, M. UNGER, « Hansische Arbeitstagung in Schwerin vom 24. bis 26.10.1956 " in : Zeitschrift für Geschichtswissenschaft, 5, 1957, n 1, p. 148-151; EID., "Hansische Forschung in der DDR» in: ibid., 4, 1956, $\mathrm{n}^{\circ} 6$, p. 1055-1056 ; M. UNGER, M. КOSSOK, K. FRITZE, «Hansische Arbeitstagung in Berlin » in : ibid., 7, 1959, n 5, p. 1108-1115; G. HEINTZ, «6. Tagung der Arbeitsgemeinschaft des Hansischen Geschichtsvereins in der DDR » in : ibid., 9, 1961, n 3, p. 652-656.

59. La présentation de ces problèmes «comme un phénomène principalement ou même exclusivement européen revient finalement à les détacher de leur ancrage dans leur histoire nationale. C'est pourquoi les historiens est-allemands de la Hanse refusent une telle conception qui soutient directement l'idéologie de l'OTAN » : K. FRITZE, E. MÜLLER-MERTENS, J. SCHILDHAUER, M. UNGER, Forschungen zur Stadt- und Hansegeschichte in der DDR, Historische Forschungen in der DDR. Analysen und Berichte. Zum XI. Internationalen Historikerkongress in Stockholm 1960, Berlin, 1960, p. 74-104, ici p. 77.

60. J. SCHILDBAUER, «Grundzüge der Geschichte der deutschen Hanse, zusammengestellt von der Arbeitsgruppe Hansegeschichte der Universität Greifwald» in: Zeitschrift für Geschichtswissenschaft, 11, 1963, $\mathrm{n}^{\circ} 4$, p. 725-746, ici p.730; E. ENGEL, «Konstituierung der Hansischen Arbeitsgemeinschaft der Deutschen Historiker-Gesellschaft » in: ibid., 19, 1971, p. 667-669. Voir à ce sujet M. MIDDELL, Weltgeschichtsschreibung, op.cit., p.946, n. 3. Pour un panorama des recherches récentes sur l'histoire de la mer Baltique dans une perspective d'histoire globale, voir A. Komslosy, H.-H. NOLTE, I. soomAn (dir.), Ostsee 700-2000. GesellschaftWirtschaft-Kultur (Edition Weltgeschichte, 11), Wien, 2008 ; en outre, de nouvelles recherches sont en cours dans l'école doctorale (Graduiertenkolleg) « Kontaktzone Mare Balticum : Fremdheit und Integration im Ostseeraum » à l'Université de Greifswald.

61. K. HAFEZ, Orientwissenschaft in der DDR. Zwischen Dogma und Anpassung, 1969-1989, Hamburg, 1995 ; U. van der Heyden, Die Afrikawissenschaften in der DDR. Eine akademische Disziplin zwischen Exotik und Exempel. Eine wissenschaftsgeschichtliche Untersuchung, Hamburg, 1999. 
62. W. MARKov, Zwiesprache mit dem Jahrhundert. Dokumentiert von Thomas Grimm, Berlin, 1989, p. 243-272.

63. Voir le résumé rétrospectif et autocritique de l'un des représentants majeurs de l'étude comparée des révolutions: М. козsок, «Im Gehäuse der selbstverschuldeten Unmündigkeit, oder: Umgang mit der Geschichte» in: Id., Ausgewählte Schriften, vol. 3 : Zwischen Reform und Revolution : Übergänge von der Universal- zur Globalgeschichte, Leipzig, 2000, p. 277-288.

64. Id., "Von der Universal- zur Globalgeschichte » in : ibid., p. 297-308 (conférence prononcée lors du colloque d'histoire globale organisé par Bruce Mazlish et Ralph Buultjens à Bellagio. Le manuel d'histoire mondiale utilisé dans presque toutes les universités: Id. (dir.), Allgemeine Geschichte der Neuzeit 1500-1917, Berlin, 1986.

65. Voir l'autobiographie de Walter Markov, dont des extraits sont publiés: W. MARKOV, Wie viele Leben hat der Mensch. Eine Autobiographie aus dem Nachlass, Leipzig 2009, notamment p. 337-360. Les contributions sont présentées dans : Actes, XII Congrès International des Sciences Historiques, Vienne, 20 Août-5 Septembre 1965, Wien, 1965 ; Mezdunarodnyi Kongress Istoriceskich Nauk 1970, Doklady kongressa, Moscou, 1973. Cette insertion internationale des historiens est-allemands et marxistes est largement occultée par A. BLÄNSDORF, «Zusammenarbeit zwischen Historikern im geteilten Europa. Das Comité international des sciences historiques und die internationalen Historikerkongresse, 1945-1990» in: A. FLEURY, L. JíLEK (dir.), Une Europe malgré tout 1945-1990. Contacts et réseaux culturels, intellectuels et scientifiques entre Européens dans la guerre froide, Bruxelles, 2009, p. 183-202.

66. Le parallélisme dans le choix des thèmes et les sources utilisées avec la situation d'alors en RDA est frappant. Pour une approche des études régionales dans les deux États allemands, voir F. BRAHMS, J. MEISSNER, “AUßereuropa“ im Spiegel allgemeiner Vorlesungsverzeichnisse. Konjunkturen der humanwissenschaftlichen Beschäftigung mit Afrika und Lateinamerika an ausgewählten Hochschulstandorten, 1925 bis $1960 »$ in : M. MIDDELL, U. THOMS, F. UEKOETTER (dir.), Verräumlichung, Vergleich, Generationalität. Dimensionen der Wissenschaftsgeschichte, Leipzig, 2004, p. 70-94.

67. Pour un témoignage important de cet effort, voir E. SCHULIN, Universalgeschichte, Köln, 1974.

68. G. HÜBINGER, J. OSTERHAMMEL, E. PELZER (dir.), Universalgeschichte und Nationalgeschichten, Freiburg, 1994.

69. Jürgen osterhAMmel a œuvré à la réception en Allemagne de l'histoire mondiale: J. OSTERHAMMEL, "Sozialgeschichte im Zivilisationsvergleich. Zur künftigen Möglichkeit komparativer Geschichtswissenschaft » in : Geschichte und Gesellschaft, 22, 1996, n², p. 143-164 ; Id., " „Höherer Wahnsinn“. Universalhistorische Denkstile im 20. Jahrhundert » in : H. W. BLANKE, F. Jäger, T. SANDKÜHLER (dir.), Dimensionen der Historik. Geschichtstheorie, Wissenschaftsgeschichte und Geschichtskultur heute. Jörn Rüsen zum 60. Geburtstag, Köln, 1998, p. 277-286 ; ID., « Die Wiederkehr des Raumes : Geopolitik, Geohistorie und historische Geographie » in : Neue Politische Literatur, 43, 1998, p. 374-397; Id., Geschichte jenseits des Nationalstaats. Studien zu Beziehungsgeschichte und Zivilisationsvergleich, Göttingen, 2001.

70. R. WENDT, Vom Kolonialismus zur Globalisierung. Europa und die Welt seit 1500, Paderborn, 2007.

71. Une comparaison avec l'URSS confirme cette pauvreté en personnel de la RFA et de la RDA; les recherches russes, après les relations scientifiques intenses des années 1950, eurent une influence durable sur l'historiographie de la RDA, mais après que cette dernière cessa d'exercer sa fonction de traduction, ces recherches furent à peine reçues en Europe occidentale.

72. M. RIEKENBERG (dir.), Geschichts- und Politikunterricht zeitgemäß ? Fragen und Bemerkungen aus der Sicht der Regionalwissenschaften, Leipzig, 2005.

73. Voir les contributions dans : M. MIDDELL (dir.), Transnationale Geschichte, op. cit.

74. Voir la liste des écoles doctorales sur le site Internet de la DFG et les projets de Graduate Schools dans le cadre de l'Exzellenzinitiative du gouvernement. Le Conseil Scientifique de la DFG a 
recommandé d'orienter les Area Studies vers l'histoire globale et de regrouper les ressources dans les différentes universités par spécialisation régionale. Il a également rendu une évaluation très positive sur les centres de recherches en sciences humaines créés après 1990 et se consacrant à l'histoire globale. Cet essor invite donc à relativiser l'échec de la nomination de spécialistes d'histoire globale à la direction de l'Institut Max-Planck d'histoire à Göttingen.

75. Le Historikertag d'Aix-la-Chapelle (1990) a retenu pour la première fois le thème : « un seul monde ", en résonance avec la section consacrée à l'histoire globale lors du CISH d'Oslo la même année. La place accordée à l'histoire globale dans les Historikertage s'est accrue depuis, tout comme l'intérêt des associations des enseignants du second degré en histoire. Voir le programme du Historikertag 2010 (« Au-delà des frontières ») à Berlin : http://www.historikertag.de.

76. Voir http://wwwback.jacobs-university.de/mgh/index.shtml.

77. Voir www.uni-leipzig.de/gesi/emgs.

78. Voir la brochure publiée en 2010 à l'occasion du vingtième anniversaire de ce type de soutien (http://www.dfg.de/download/pdf/dfg_im_profil/geschaeftsstelle/

publikationen/20_jahre_graduiertenkollegs.pdf).

79. Précisons ici que les écoles doctorales et les Excellenzcluster formés dans le cadre de l'ExcellenzInitative ne sont pas pris en compte.

80. Voir www.die-gdi.de.

81. Voir www.giga-hamburg.de.

82. Voir www.swp-berlin.org.

83. Voir U. FREITAG, A. VON OPPEN, "Translokalität als ein Zugang zur Geschichte globaler Verflechtungen » in : http://hsozkult.geschichte.hu-berlin.de/forum/ 2005-06-001.

84. Parmi ces centres, le Zentrum für Zeithistorische Forschungen s'est longtemps centré sur l'étude la RDA, mais entreprend depuis quelques années des comparaisons avec l'Europe orientale et l'URSS. Le Geisteswissenschaftliche Zentrum für Geschichte und Kultur Ostmitteleuropas à Leipzig est depuis longtemps actif dans l'étude comparée avec les voisins (sud-)orientaux de l'Allemagne. Ces centres recourent au concept de globalisation, tout comme les institutions spécialisées sur l'Europe de l'Est. Le Zentrum Moderner Orient s'intéresse aux relations entre sociétés musulmanes et occidentales et s'inscrit donc pleinement dans les débats et recherches sur la globalisation.

85. B. STUChTEY, E. FUCHS (dir.), Writing World History, op. cit. ; Eid. (dir.), Across Cultural Borders : Historiography in a Global Perspective, Lanham, 2002.

86. Voir http://www.ghi-dc.org.

87. Voir http://www.sfb536.mwn.de/.

88. Ibid. ; voir aussi U. BECK, A. GIDDENS, S. LASH, Reflexive Modernisierung - Eine Debatte, Frankfurt am Main, 1996 ; ead. (dir.), Politik der Globalisierung, Frankfurt am Main, 1998.

89. Voir http://www.sfb597.uni-bremen.de/pages/welcome.php?SPRACHE=de.

90. Important: M. zÜRN, Regieren jenseits des Nationalstaates: Globalisierung und Denationalisierung als Chance, Franfurt am Main, 1998. Voir aussi L. BROCK, M. ALBERT, « Entgrenzung der Staatenwelt. Zur Analyse weltgesellschaftlicher Entwicklungstendenzen» in: Zeitschrift für Internationale Beziehungen, 2, 1995, p. 259-285. Pour une position opposée, voir P. EVANS, "The Eclipse of the State? Reflections on Stateness in an Era of Globalization " in: World Politics, 50, 1997, $\mathrm{n}^{\circ} 1$, p. 62-87. Les postulats du SFB 597 ont d'abord été affirmés par K. OHMAE, The End of the Nation State : The Rise of Regional Economies, New York, 1995.

91. Voir http://www.sfb-governance.de/.

92. Voir T. RISSE, U. LEHMKUHL (dir.) Regieren ohne Staat? Governance in Räumen begrenzter Staatlichkeit, Baden-Baden, 2007.

93. Voir http://www.sfbtr15.de/index.php.

94. Voir http://www.foev-speyer.de/governance/inhalte/01_home.asp. 
95. Voir http://www.geschkult.fu-berlin.de/e/oas/japanologie/forschung/ Akteure_kulturelle_Globalisierung/index.html.

96. N. Luhmann, Soziale Systeme: Grundriß einer allgemeinen Theorie, Frankfurt am Main, 1984.

97. Voir http://www.uni-bielefeld.de/(de)/soz/iw/graduiertenkolleg/.

98. Voir http://www.uni-leipzig.de/ral/gchuman/index.php? option=com_content\&task=view\&id=11\&Itemid=9.

99. M. MIDDELL, K. NAUMANN, « Global History ... », op. cit.

100. Voir http://www.uni-giessen.de/gkmedienereignisse/home/index.php.

101. Voir F. BÖSCH, N. FREI (dir.) Medialisierung und Demokratie im 20. Jahrhundert, Göttingen, 2006.

102. C. LEGGEWIE, Die Globalisierung und ihre Gegner, München, 2003 ; F. CRIVELLARI, M. SANDL, « Die Medialität der Geschichte. Forschungsstand und Perspektiven einer interdisziplinären Zusammenarbeit von Geschichts- und Medienwissenschaften » in: Historische Zeitschrift, 277, 2003, p. 619-654.

103. Voir http://www.uni-mainz.de/forschung/19598.php (14.09.2008).

104. Voir http://www.kuwi.euv-frankfurt-o.de/de/dekanat/startsite_news/news6/ kolleg_transnationale_raeume.pdf. Sous le même intitulé, le Bauhaus Kolleg VI (Dessau) a étudié en 2004-205 la naissance d'opinions publiques transnationales dans les métropoles (http:// www.bauhaus-dessau.de/index.php?bauhaus-kolleg-vi).

105. C'est dans une direction analogue que travaillent un groupe au Geisteswissenschaftlichen Zentrum Geschichte und Kultur Ostmitteleuropas (GWZO, Leipzig) sur «l'Ostmitteleuropa: une approche transnationale", et le Herder-Institut (Marbourg) et jusque récemment le collège doctoral sur l'histoire comparée de l'Europe (Berlin). Pour l'inclusion de l'Ostmitteleuropa dans l'histoire européenne: J. коскA, «Das östliche Mitteleuropa als Herausforderung für eine vergleichende Geschichte Europas " in: Zeitschrift für Ostmitteleuropa-Forschung, 49, 2000, p. 159-174.

106. Voir http://www.uni-rostock.de/andere/grk1242/.

107. Voir http://www.geschundkunstgesch.tu-berlin.de/fachgebiet_neuere_geschichte/menue/ dfg_graduiertenkolleg/parameter/de/.

108. Voir http://www.grakov-berlin.de. Le Max-Planck-Institut für ausländisches öffentliches Recht (Heidelberg) étudie avant tout le droit des organisations internationales, en particulier celles liées à l'ONU, le droit économique international, le droit international de l'environnement et le droit des espaces communautaires internationaux (Arctique, Antarctique, espace). Voir http:// www.mpg.de/instituteProjekteEinrichtungen/institutsauswahl/voelkerrecht/index.html.

109. Voir http://www.uni-tuebingen.de/gk.globale-herausforderungen/html/allge meines.htm.

110. Voir http://www.geisteswissenschaften.fu-berlin.de/v/interart/.

111. Voir http://www.social-globalization.uni-kassel.de/.

112. Voir http://www.phil.uni-mannheim.de/pk_globalisierung.

113. Voir http://www.uni-leipzig.de/ral/gchuman/index.php? option=com_content\&task=view\&id=13\&Itemid=43.

114. L'Edition Weltregionen (éditions Promedia à Vienne) consacre un volume à chaque région et réunit pour cela dix à quinze spécialistes en histoire et sciences de la culture. Les derniers volumes publiés, sur l'Océanie et l'Océan Indien, tiennent compte des critiques sur le danger d'une essentialisation des continents.

115. La série "Expansion-Interaktion-Akkulturation» est publiée chez l'éditeur viennois Mandelbaum et a le même ancrage institutionnel que la série précédemment mentionnée, même si elle suit une approche centrée sur l'analyse des interactions plus que sur la comparaison. Dans le même contexte est née la série "Historische Sozialkunde/ Internationale Entwicklung" (aux éditions Promedia et Südwind) qui s'intéresse surtout aux évolutions internationales actuelles. Elle a publié plus de trente volumes, sur les relations Nord-Sud, les flux mondiaux de 
marchandises ou l'histoire des migrations, qui ont une importance certaine pour la recherche en histoire globale allemande.

116. P. FELDBAUER, B. HAUSBERGER, J.-P. LEHNERS (dir.), Globalgeschichte der Welt 1000-2000 (Essen), Wien (depuis 2008).

117. Voir J. OSTERHAMmEL, «Aufstieg und Fall der neuzeitlichen Sklaverei. Oder: Was ist ein weltgeschichtliches Problem ? » in : ID., Geschichtswissenschaft jenseits des Nationalstaats, Göttingen, 2001, p. 342-369.

118. J. OSterhammel, N. P. Petersson, Geschichte der Globalisierung, Dimensionen-Prozesse-Epochen, München, 2003.

119. J. osterhammel, Die Verwandlung der Welt. Eine Geschichte des 19. Jahrhunderts, München, 2009.

120. H.-H. NOLTE, Weltgeschichte: Imperien, Religionen und Systeme, 15.-19. Jahrhundert, Köln/ Weimar/ Wien, 2005 ; Id., Weltgeschichte des 20. Jahrhunderts, Köln/ Weimar/ Wien, 2009.

121. A. JOCKENHÖVel, W. DEMEL (dir.), Grundlagen der globalen Welt. Vom Beginn bis 1200 v.Chr., Darmstadt (WBG-Weltgeschichte, I), 2009.

122. W. DEMEL (dir.), Entdeckungen und neue Ordnungen. 1200 bis 1800, Darmstadt, 2010 (WBG Weltgeschichte, IV).

123. D. DINER, Das Jahrhundert verstehen. Eine universalhistorische Deutung, München, 1999.

124. Pour en citer quelques-uns: S. Conrad, S. Randeria (dir.), Jenseits des Eurozentrismus. Postkoloniale Perspektiven in den Geschichts- und Kulturwissenschaften, Frankfurt a. M./ New York, 2002 ; M. Grandner, D. Rothermund, W. Schwentker (dir.), Globalisierung und Globalgeschichte, Wien, 2005 ; B. Schäbler (dir.), Area Studies und die Welt. Weltregionen und neue Globalgeschichte, Wien, 2007 ; S. Conrad, A. Eckert, U. Freitag (dir.), Globalgeschichte. Theorien, Ansätze, Themen, Frankfurt a. M./ New York, 2007 (début d'une série chez l'éditeur Campus, plusieurs monographies publiées depuis) ; J. Osterhammel (dir.), Weltgeschichte, Stuttgart, 2008 ; M. Middell, U. Engel (dir.), Theoretiker der Globalisierung, Leipzig, 2010.

\section{AUTEURS}

\section{MATTHIAS MIDDELL}

Matthias Middell est directeur du Global and European Studies Institut de l'Université de Leipzig.

\section{KATJA NAUMANN}

Katja Naumann est chargée de recherche au Geisteswissenschaftliches Zentrum Geschichte und Kultur Ostmitteleuropas de l'Université de Leipzig. 